\title{
Incongruent Counterparts and The Intuitive Nature of Space
}

A. T. Winterbourne

Birmingham Polytechnic

Kant's views on space and time changed during his life, and reflected at various times his pre-occupation with Newtonianism, and Leibnizian relationalism, before reaching maturity in the doctrine of transcendental idealism. His discovery of the synthetic a priori nature of geometrical propositions made some radical transformation of these earlier views necessary, although Kant had already recognised their inadequacies before the critical synthesis had been accomplished. The conflicting pulls of Newton and Leibniz--neither of whose theories were ever totally abandoned--and the concern with the false dichotomy of logic and experience, each played their part in shaping the critical theory of space and time as presuppositional forms. The history of Kant's thinking on space in particular is highliglited by the argument from incongruent counterparts, which seemed to occupy Kant as a problem, though not always as a paradox, throughout the vicissitudes of his thinking on space. More important, the argument is, at the very least, a supplementary proof of the a priori and intuitive nature of space."

The argument first appears in the article "Concerning the Ultimate Ground for the Differentiation of Regions in Space". which appeared in 1768.2 In this paper, Kant seems to be moved to write under the stimulus of the Newton-Leibniz debate. He argues against those 'German philosophers' who deny reality to space, and at this stage supports Newton. There is though, much more to it than this. Even though the original article was a defense of an absolutist position, it nonetheless contains most of the important insights and considerations which were to lead eventually to a rejection of its most explicit conclusion, viz.. the existence of Newtonian space, and which developed into the critical view of space as a priori intuition and as transcendentally ideal. Kant is concerned with the grounds for differentiating 'regions' in space. For Newton, space was a 'substantial' entity; for Leibniz, spatial qualities were reducible to the properties of phenomenal aggregates which are not in themselves ultimately real. The stimulus of this de- 
bate leads Kant to suggest a justification for an antiLeibnizian position, and an indirect confirmation of the Newtonian alternative. The argument is not an explicit attempt to prove that space is absolute and not relational, but is more plausibly regarded as one that favours the view that space is some kind of reality. rather than a merely ideal system of relations. For Kant, the notion of 'differentiable regions' implies that space is real.' Kant will argue that a full explanation of incongruent counterparts entails reference both to the objects, and their relations in space. regarded as distinct from those objects. That the parts of space are only possible through limitations of a prior totality of space, is an assertion that Kant makes in all his writings, both pre-critical and critical; it is a view that is certainly found in Newton. The positions of the parts of space presuppose a region, according to which they are ordered; that is, there is some ordering relation through which all possible positions in space are connected. At this stage it seems clear that the whole of which these parts are parts, is absolute space, although Kant does not simply assume this to be the case. A region, he says, does not consist in the relations that one thing in space has to the next--this would be position, or 'relation of the situation'. For Leibniz, the region must consist phenomenally in relations obtaining between substances--by means of confused perception--and cannot refer to any external space in which the relations are possible. Kant asserts that a region is the relation of a system of positions to absolute space. Now the position of the parts of an object with respect to each other are sufficiently recognised from the object itself. The region to which this order of the parts is directed, is related to absolute space: the region, in this sense, corresponds to Newton's 'relative' space, and because of the definition of spatial totality in terms of limitations of relative spaces, this region 'belongs' to absolute space. It is Kant's intention to offer grounds for the assertion of the independent reality of this system of all possible regions, i.e., absolute space. Such a demonstration would be Impossible, Kant says, if appeal is made merely to abstract metaphysical principles; this challenges Leibniz's attempt to base his refutation of Newtonian space on the principle of sufficient reason etc.

since space has three dimensions, three surfaces can be conceived in physical space. We have, says Kant, knowledge of external things only by means of relations of sensuous impressions to ourselves: from the relation of these intersecting surfaces to our body we derive the ultimate foundation for constructing the concept of spatial regions. This is a clue to understanding Kant's later view of space as intersubjective, and will be taken up again below. 
Kant is trying to show that the complete principle of determining physical form does not, and cannot rest merely on the relation and situation of the parts of objects with respect to each other, but must also rest on their relation to external space. This formulation of the position suggests very strongly that Kant never intended to demonstrate that the difference between incongruent counterparts depends purely on the relation to space, but requires in addition reference to the shape of the object--that is, internal characteristics. This relation to absolute space cannot be immediately 'perceived', but the physical differences that rest uniquely and alone on this ground can be perceived. Such differences are of course the left- and righthandedness of certain objects.

Consider a left and right hand: in terms of geometrical properties, proportions, situation of parts relative to other parts, etc.. a complete description of one serves as a complete description of the other. "So much may be sufficient to understand the possibility of completely like and similar and yet incongruent spaces." " Now Kant can only mean by 'spaces' here, the region that a body occupies, or the containing surface of the body. This is important, since Kant's most interesting solution to this problem will concern the relationship holding between a body and the external space in which the body exists. Kant's next point is confusing. He says that the determinations of space are not consequences of the situations of the parts of matter relative to each other: rather, the situations of the parts are consequences of the determinations of space. Yet the parts of an object are supposediy describable without reference to external space; I take this to accord with the Leibnizian claim that these relations are not properly describable as spatial at al1. Here however Kant is saying that the situations of the parts are somehow to be considered as consequences of the determinations of space. Yet regions, on Kant's view, can be differentiated only through the connections that objects have to absolute space. It is difficult to see what is involved in the assertion that space as such could have determinations which were not simply relations to already existing objects, unless one presupposes a Newtonian spatial structure of differentiated points, which 'exist' prior to and independently of physical objects."

After consideration of the geometrical properties of the two objects, it is clear that some difference remains unexplained; this difference, Kant insists, must be internal. The surface which encloses one cannot enclose the other. The fact that this limiting space' can be applied in one case and not the other suggests that the difference rests on some 'inner principle': However, it cannot have anything to do with how the parts of the object are connected: first- 
1y, because different regions cannot be explained in this way; and secondly, because so far as the arrangement of the parts is concerned, the objects are identical. Kant says that in the constitution of bodies real differences are found which are connected with absolute and 'original' space, since it is only through this space that the relation obtaining between physical things is possible.

At the end of the 1768 article Kant makes some remarks about space which anticipate the position he was eventually to take in his mature philosophy; they also relate to some otherwise puzzling remarks in the 1768 article, and to the use he subsequently made of the argument in later writings. Absolute space, he concludes, is not an object of external sensation: it is a fundamental concept which makes these sensations themselves possible. This suggests Kant's mature position that takes space as a necessary representation underlying all outer impressions of the senses, i.e. an a priori intuition. Kant considered that all knowledge of external things by means of relations of sense impressions must ultimately be related to a perceiving subject. I take this to mean that without having seen the full implications of the view at this stage, Kant has seen the impossibility of regarding space as a purely intellectual entity, which might be described without reference either to objects, which differentiate it, or to perceiving subjects, which essentialiy constitute it. It is only by means of the relations of bodies that we can 'perceive' the relations to 'pure' space."

There is a short reference to incongruent counterparts in the Inaugural Dissertation of 1770 . Here, the use of the argument occurs within the wider context of arguments designed to show that space and time are not rational entities, nor objective ideas, but are in fact 'phenomena'. Whereas in the earlier article it seemed sometimes that Kant was wavering between Leibnizian rationalism and Newtonianism seen in relation to a whole system of physics, the Dissertation argument firmly rejects both of these in favour of a recognisably subjectivist approach, which would develop into transcendental idealism. All the important new principles of the critical position are embryonically present in the Inaugural Dissertation. The concept of space is a singular representation, and is pure intuition; that is, space is an a priori particular. The argument from incongruent counterparts supports this position, which I suggested is latent, and unrecognised in the earlier article. With opposite handed objects the diversity, that is, the 'discongruity', can only be noticed by a certain 'act' in pure intuition. In the 1768 article the solution to the problem was that the objects differed in their relations to external space; this position has now been abandoned in favour of the 
far more subtle view which leads to transcendental idealism. However, the solution offered in the Dissertation might be considered on other grounds. 7 What I take kant to be saying here is this: any handed object, when considered apart from the conditions of experience, is indeterminate with respect to whether it is left or right. I interpret Kant here to be making a transcendental idealist point in the restricted sense in which this implies some doctrine about meaning. A single hand, alone in the universe, is not an object of a possible experience, and as such it is literally meaningless to say that such a hand is determinate with respect to whether it is left or right. These latter concepts presuppose an orientation in space; since space is a form of representation and not an objective inclependently existing entity, such orientations presuppose a perceiving mind. There is a definite Leibnizian legacy here. 'Left', 'right'. 'large'. and 'small' are treated as relational; they require some given franework in order to be fully intelligible. kant is adding the subjectivity of space as the standard against which such concepts attain significance. For Leibniz the relations would be ultimately explicable in terms of the attributes of monads: Kant is trying to show that this explanation leaves out someting important--hence his assertion that the diversity of the hands can only be noticed in an act in pure intuition. Ideas like left and right are merely 'conventional', and cannot be given a meaning--a sense in abstraction from perceiving minds."

The transcendental idealist interpretation of the argument is naturally supported by the use made of it in the Prolegomena. There Kant argues against those who take space to be a real quality of things in themselves. This complements the argument in the Dissertation, which takes space to be a pure intuition. Kant points out that various figures show, in spite of complete 'inner agreement', an outer relation, which makes it impossible to superpose one figure on the space of the other; yet the difference is given as being inner. This inner difference, which conceptual understanding cannot define, reveals itself through the outer relations to space. What I think Kant means here is that this difference only shows itself through the relation it has to an experiencing subject, for whom space is the condition of the possibility of any and every object in space. Consider opposite hands: there are no inner differences that can be revealed through merely conceptual analysis; yet the differences are inner so far as the senses tell us. In other words, while the objects are similar when considered conceptually, they are experienced as being essentially different. Since we can have no experience of space as an independent self-subsisting entity. then the difference must be grounded in us, through space as 
representation. Kant's solution in the Prolegomena is that since objects are not representations of things as they are in themselves, and as a pure understanding would cognise them--in an 'intellectual intuition'--but rather, are sensible intuitions, that is, appearances, the possibility of which rests on the relations of certain things unknown in themselves to sensibility: then the difference between incongruent counterparts can only be made intelligible by referring them to an act. which takes place in pure intuition."

There seem to be three main lines through which to approach Kant's problem. First, there is the insight of the original 1768 article, in which the 'solution' involves the postulation of something like Newtonian space; second, there is the transcendental idealist solution, suggested in the Dissertation, and then developed in the Critique itself, (although there is no mention of the problem in the latter); and third, there is the modern approach, which can be traced to both Leibniz and Kant, and relies upon either abstract geometrical considerations, or a more general appeal to the topological features of objects and their relations to the spaces in which they are embedded. In a recent work. Nerlich has claimed that Kant's original ideas were almost entirely correct. ${ }^{10}$ Nerlich generalises the problem by referring the argument to 'enantiomorphic' objects--spirals, hands, etc...withoug bringing in the question whether it is feasible to consider the determinateness of such objects. What is at stake is the handedness of the object, rather than whether it is a left or a right hand. Nerlich claims that when Kant introduced the handless body into his thought-experiment, he was aiming to show that the object must have been an enantiomorph, not that the hand must have been either left or right. Nerlich says, following Kant, that whether an object is an enantiomorph or not depends on the nature of the space it is in, thus confirming Kant's original insight. Thus the problem of incongruent counterparts depends for its resolution on considerations of both the shape of the object and the 'shape' of space. This is the point that I attributed to kant above. Kant says that a complete principle of determining physical form does not rest merely on the relation and situation of the parts of the object with respect to each other, but also on its relation to external space. Nerlich develops this idea, employing the ideas of 'orientable' and 'nonorientable' spaces to demonstrate how the relationship between the object, and the kind of space in which it is 'embedded', determines whether it is enantiomorphic or, as he puts it, 'homomorphic'. Objects are homomorphic if they are indifferent with respect to leftness or rightness; for example, opposite-handed triangles in a one-sided non-orientable manifold such as a Klein 'bottle', can be reversed by transporting them through 
the space by means of a continuous motion." Now Nerlich claims that it is senseless to say that a hand is neither enantiomorphic nor homomorphic: this is a direct challenge to a transcendental idealist interpretation of the argument, which in effect states that the question of whether the lone hand is either left or right is meaningless before this is taken in the context of possible experience. Nerlich's point is more difficult. It is one thing to claim that the question as to which hand it is must be meaningless, but it is quite another to say that it is also meaningless to assert that it is handed as such. This brings us to guestions of topology, and some modern 'solutions' to this whole problem.

There is one way of dealing with Kant's original problem which seems both elegant and compeliling, and yet takes the argument into a thicket of epistemological difficulties. The argument can be put like this: Kant was puzzled by how it is that two objects can be alike in essential conceptual characteristics and yet manifest an obvious dissimilarity. However, if we take the problein down one spatial dimension, the 'paradox' admits of a clear and intuitively satisfactory solution. Consider two opposite handed shapes in two dimensions, and how any two dimensional 'beings' living in such a universe, (e.g. 'beings' whose space was confined to the surface of a sphere) would react to such objects. They would be, so the argument goes, as puzzled by these shapes as we are by enantiomorphic objects in three dimensions. Yet we can see that their puzzlement is simply due to the limitations of spatial imagination. By rotating one of the two-dimensional objects in our 'extra' spatial dimension, we can superpose one onto the space of the other. The twodimensional beings would have no way of visualising the process that had occurred, at least not as a continuous transformation of the object through three dimensions; but if--and here the analogy becomes a fairy-tale--they were sufficiently clever mathematicians, they might be able to work out the properties of a space possessing one more dimension than their own, and thus explain the existece of incongruent counterparts in two-dimensional space. Thus it is easy to see why we are so puzzled by such objects in three dimensions: the 'solution' is to 'rotate' one of them through a space of four dimensions; we could then superpose it on the space of its counterpart.

This analogical solution raises almost as many problems as it was designed to solve. The first point is very general, and is aimed at all wild extrapolations from concepts amenable to mathematical manipulation--ndimensional spaces, for instance (when $n=3$ )--to actual possibilities: in other words, the move from what is logically possible, to what is really possible, where the latter concerns those things that could be 
part of a possible experience. ${ }^{12}$ There are not, and could not be, beings of two-dimensions who, in addition to having two-dimensional sense organs, are also capable of perceiving two-dimensional plane figures, and have two-dimensional brains that think mathematically. The second point is less frivolous, but is implied by the first: possible worlds of one, two or $3+n$ dimensions are abstractions--not real, but ideal. We should no more think of 'hypercubes' as real entities, than we should hypostatise points, lines and surfaces in Euclidean space. Such objects 'exist' only insofar as there exist mathematical operations and relations incorporating them. There is no reason to believe in a physical space of four dimensions: the argument above therefore remains an analogy. which theoretically accounts for two-dimensional incongruent counterparts, but which cannot be simply extended to higher dimensions. The problem in three dimensions concerns the application of abstract geometrical principles to a particular spatio-temporal phenomenon; the analogical argument concerns the application of one abstraction to another. The purely theoretical nature of this analogical solution may in addition be taken as confirmation of the necessity of the three-dimensional character of perceptual space. We can say that the perceptual space of any being will have the same number of dimensions as opposite handed objects which cannot be superposed by continuous transformations of the objects. Thus our perceptual space has three dimensions, since there do not exist for us incongruent counterparts of twodimensions; it remains logically possible that our three-dimensional space is the 'surface' for some $3+n$ dimensional being; but our perceptual apparatus does not allow us to witness the 'rotation' of a threedimensional solid in any higher space as a continuous motion.

There is an additional consideration that casts doubt on the plausibility of the two-dimensional analogue. The latter suggests that handedness is determined by some putative relation to a higher dimensional space, but there are reasons for thinking that it depends, as Nerlich indicates, on the way the object is 'entered' into its own space. Consider a left-glove shape, in two-dimensions, living on the curious surface of a Mobius band--a non-orientable space having only one side. Such an 'object' is homomorphic, since transporting it around the space will turn it into a right hand. However, unless our three dimensional space can be plausibly considered to be similarly nonorientable, the equivalent process in three dimensions could not be carried through. The two ways of resolving the problem by analogies with other spaces leave this alternative: either enantiomorphic objects are really homomorphic--because our space is a nonorientable manifold; or we just have to believe in a 
real fourth spatial dimension. In either case the original 'paradox' dissolves, but the mysteries deepen in other areas. It has been suggested that if some of the laws of physics are not left-right indifferent, then it should be possible to say of some object that it is left- or right-handed even if it was, like 'Kant's hand', alone in the universe."' Since time is one-dimensional, there can be no temporal 'mirror images', in the same way that there are no mirror images for lines in two-dimensional space. But there is a further consideration: space has no preferred direction; if it had, then left- and right-handedness could be uniquely determined--rather in the way that the mathematician deals with it in terms of even and odd permutations, except that in such a case the attribution of direction would be non-arbitrary. What Kant has pointed out is that two opposite handed objects can be seen to be such, but not defined. The direction of time always gives us a unique difference between before and after: the lack of intrinsic direction for space presents us with the problem of mirror images, which may be directly intuited, but conceptually defined only by an arbitrary act of pointing that presupposes this intuition.

The three attempts to resolve the problem--that is, by reference to external space; by appeal to the nature of space as representation; and through topological analysis, all add to an understanding of the original problem, operating as they do on different levels of philosophical abstraction. There is some support for Nerlich's solution, (appealing to the fact that space itself has a structure) from Hermann Weyl. The congruent mappings of space from the group of transformations called the Euclidean group of motions. Once this group is known, congruent volumes may be defined as portions of space that can be carried into each other by continuous transformations. The facts suggest, according to Weyl, an interpretation according to which the group of Eucliclean motions of congruent mappings expresses an intrinsic structure 'stamped by space on all its objects'. However, as Weyl points out, the requirement of continuity eliminates transformations of signature minus: ". . . a rigid body could go over into its mirror image only by a discontinuous jump. . Kant finds the clue to the riddle of left and right in transcendental idealism: the mathematician sees belind it the combinatorial fact of the distinction of even and odd permutations."1" This presupposes an independent definition of even and odd; the mathematician--legitimately--redefines left and right as - and +; he substitutes one convention for another. Unless we had a plior intuitive understanding of the difference between mirror images--unless, that is, we could recognise them as different, we have no basis on which to label them - and +; Weyl accepts this 
elsewhere, when he remarks that "... to the scientific mind there is no inner difference between left and right. It requires an arbitrary act of choice."Is Weyl asks us to consider a thought-experiment like Kant's: consider God's creative act; had God, rather than making first a left and then a right hand, begun by making a right one, then He would have changed the plan of the universe not in the first but in the second act, by bringing forth a hand which was equally rather than oppositely oriented to the first one. This seems to me to be part of Kant's key insight in the 1768 article, even if he did not fully recognise it himself. 16

I mentioned above that Kant's 1768 article was concerned not only with offering indirect support to a Newtonian view of space, but also with a direct challenge to Leibniz, and in particular, the principle of the identity of indiscernibles. As a conclusion to this paper, I will take up this general point very briefly.

How could Leibniz explain the existence of incongruent counterparts? To some extent, the discussion just undertaken has suggested how a relational view of space assists in our understanding of the problem; but there is in addition a problem for Leibniz which operates on and between two levels of Lelbniz's system: that is, phenomenal aggregates and ultimately real substances. The identity of indiscernibles implies that no two individuals can be exactly alike in all their predicates, and differ solely in respect of spatio-temporal location. This applies to substances-complete individual monadic histories. The distinguishability of substances is referrable, ultimately. to the infinite mind of God, who knows the complete notions of all substances. Why does this present a problem for Leibniz? The difficulty over incongruent counterparts seem irrelevant here, since it is obvious that we are confronted with two different objects--and this is the problem. The point I think is that when we consider the complete notions of two such objects, we have seen above that there is difficulty in accepting that there is an inner difference; they are alike in all essential, differentiable properties. There is no internal property possessed by one which is not possessed by the other. On Leibniz's principle, they should be the same object. He may not differentiate the objects by reference to relations in external space, because there is no such external space for the objects to be related to, in a way which would guarantee their uniqueness." Spatial relations are conseguences--phenomenal consequences---of the perceptions of non-spatial monads. There is an obvious difference between two objects which are counterparts, and the principle of the identity of indiscernibles seems, superficially, untouched, since this is precisely what it asserts, viz., that no two objects are incapable of 
being differentiated by means of internal properties. Yet the principle operates on the level of Leibnizian ultimate reality: the 'predicate in notion' principle seems to entail that there must be a predicate possessed by one of the objects which is absent from the complete notion of the other. Yet Kant pointed out the difficulty of explicating incongruent counterparts by means of what he would call 'merely conceptual' characteristics. For God, who reguires no spatial intuition, there can be no difference between left and right, or past and future. Thus the difference is due either to the relations that objects have to other objects: this would commit Leibniz to the view that the difference is merely phenomenal--but in what could it be well-founded? The other possibility is for Leibniz to concede that the difference is due to some property of space--'imposing its structure on objects', as Weyl puts it. This suggests that space, qua determinate system of metric relations is 'conventional', or ideal for Leibuiz, and is 'imposed' on phenomenal aggregates. Leibniz would probably have been much interested in modern views which suggest that there may be physical laws which are not left-right indifferent. There are problems with this idea, one of which is making it intelligible independently of an already presupposed framework; in addition, we can ask for some convincing account of why the perceptual, macroscopic phenomenon of handedness, should be explicable by reference to the intrinsic 'handedness' of the elements of such objects. In the same way that it is plausible to postulate micro-physical discontinuity. while accepting the necessity of phenomenal continuity, it is also quite possible that left- and right-handedness--as a philosophical problem, is a phenomenal puzzle, as Kant thought, and cannot be reduced to some putative physical differences on the microscopic or sub-microscopic level. For Kant, the problem of incongruent counterparts is that it is puzzling how two objects, with identical descriptions, can yet be intuited as obviously different; for Leibniz, the puzzle is that two objects can have identical descriptions without being the same object.

\section{NOTES}

'There is a considerable range of acceptance of this problem in Kantian commentaries. The omission of the argument from the Critique of Pure Reason is certainly puzzling: Buchdahl, in Metaphysics and the Philosophy of Science (Blackwe11, 1969), thinks it was omitted because it offers only supporting evidence for 
the a priori nature of space, and is, in any case, an analogy exemplifying what Kant thinks he has proven elsewhere; (cf. Buchdahl, p. 559) Broad places it more centrally in Kant's general theory of space: see C. D. Broad. Kant, An Introduction (Cambridge University Press, 1978), p. 37. My Own sympathies are with Ralph Walker, who takes the argument to be Kant's 'real' reason for holding space to be an intuition; cf. Walker, Kant (Routledge, 1978), p. 44.

In Kant: Selected Pre-critical Writings, ed. D. Walford and G. Kerferd (Manchester University Press, 1968), p. 36, Körner has the rather eccentric translation of 'Gegenden' as 'directions', although this will turn out to be rather appropriate at some points in Kant's discussion: See stephan Korner. Kant (Penguin, $1955)$, p. 33 .

'On the Leibnizian view incongruent counterparts must be explained by means of internal or 'purely conceptual' characteristics.

"Kant, op.cit., p. 42; my italics. cf. Gerhard Frey, Erkenntais der Wirklichkeit (Kohlhammer, Stuttgart, 1965), esp. p. 112-113.

"II. - region is related to space in general as a unity, of which each extension must be regarded as a part." Kant, op.cit.. p. 37.

'Kant concludes the 1768 article on another antiLeibnizian note by asserting that space is not a mere 'Gedankending' or an ens rationis. This terminology reappears in the Amphiboly of Concepts of Reflection in the Critique of Pure Reason, when Kant repeats this point, adding the view that 'pure' space and time are best regarded as ens imaginarium. Critique of Pure Reason, tr. N. K. Smith (MacMillan, 1970̄), p. 295-296.

'In the earlier paper, Kant had suggested a provocative thought-experiment to illustrate his point. Imagine that the universe is empty except for a single hand. Is it a left-hand, or a right-hand? Given the conceptual indeterminacy of handedness, it does not seem to make sense to assert that it is either one or the other: but now imagine that a handless body is created. Now, it seems, the hand must fit on either the left or the right wrist. Yet if it does fit on the left wrist, then it must have been a left hand before the introduction of the handless body. This shows, Kant suggested, that some 'absolute' spatial framework-a kind of 'ideal lattice' must exist independently of objects in space. Kant seems to have recognised the mistake in his reasoning here by the time he came to write the Dissertation. 
-Leibniz had already pointed this out in the debate with Clarke, in his denial of the significance of the idea that east and west might, on a global scale, be reversed. Leibniz's Third Faper, in The Leibniz-Clarke Correspondence, ed. by H. G. Alexander (Manchester University Press, 1956), p. 26. Such a change would be quite without significance; taking a Leibnizian verificationist stand, the lone hand is not merely indeterminate with respect to leftness or rightness; it is also neither large nor small.

'See Kant, Prolegomena, tr. P. G. Lucas (Manchester University Press, 1966), \$13, p. 41ff.

"See G. Nerlich, The Shape of Space (Cambridge University Press, 1976).

"An orientable space is one in which a global definition of left and right can be made; orientability coresponds to two-sidedness and non-orientability to one-sidedness; they are intrinsic topological properties independent of any external space.

${ }^{12}$ This point relates to Kant's theory of geometry. According to Kant, other geometries are logically possible, but not capable of intuitive construction in our space. It should also be noted, with reference to the 'rotation' of two-dimensional objects in ' 3 - space'. that one cannot, in any non-metaphorical sense, 'turn over' a two-dimensional object, which has only one side.

13The non-conservation of parity suggests a way of uniquely determining handedness. Since this is impossible in a non-orientable space, physical space cannot be non-orientable. It is possible to consider temporal equivalents of incongruent counterparts--or more accurately spatio-temporal equivalents, e.g., as space-time vectors in some theoreticaliy possible space-time systems. See J. Earmen, "Kant, Incongruent Counterparts and the Nature of Space and Space-time," Ratio, v. 13, (1971).

${ }^{14} \mathrm{H}$. Weyl, Philosophy of Mathematics and Natural Science (Princeton, 1949), p. 84 .

\footnotetext{
${ }^{15} \mathrm{H}$. Weyl, Symmetry (Princeton, 1952), p. 17.

ccf. Broad, op.cit., p. 42.

17 "Besides the difference of time and place there must always be an internal principle of distinction, and although there are several things of the same kind, it is nevertheless true that none of them are ever perfectly alike. Thus although time and place
} 
(external relation) enables us to distinguish things, which we do not readily distinguish by themselves, the tlings are nonetheless distinguishable in themselves. The exact determination of identity and diversity is not a matter of time and place, although it is true that the diversity of things is accompanied by time and place; because (they) bring with them different impressions about the thing." Die philosophischen Schriften von G. E. Leibniz, herausgegeben von C. J. Gerhardt, Berlin, 1875-90, v. 213. 